\title{
Effects of cell seeding density on real-time monitoring of anti-proliferative effects of transient gene silencing
}

\author{
Cigdem Selli ${ }^{1,2^{*}} \mathbb{D}$, Yasemin $\operatorname{Erac}^{2}$ and Metiner Tosun ${ }^{2,3}$
}

\begin{abstract}
Background: Real-time cellular analysis systems enable impedance-based label-free and dynamic monitoring of various cellular events such as proliferation. In this study, we describe the effects of initial cell seeding density on the anti-proliferative effects of transient gene silencing monitored via real-time cellular analysis. We monitored the realtime changes in proliferation of Huh7 hepatocellular carcinoma and A7r5 vascular smooth muscle cells with different initial seeding densities following transient receptor potential canonical 1 (TRPC1) silencing using xCELLigence system. Huh7 and A7r5 cells were seeded on E-plate 96 at 10,000, 5000, 1250 and 5000, 2500 cells well $^{-1}$, respectively, following silencing vector transfection. The inhibitory effects of transient silencing on cell proliferation monitored every 30 min for $72 \mathrm{~h}$.

Results: TRPC1 silencing did not inhibit the proliferation rates of Huh7 cells at 10,000 cells well ${ }^{-1}$ seeding density. However, a significant anti-proliferative effect was observed at 1250 cells well $^{-1}$ density at each time point throughout $72 \mathrm{~h}$. Furthermore, significant inhibitory effects on A7r 5 proliferation were observed at both 5000 and 2500 cells well $^{-1}$ for $72 \mathrm{~h}$.

Conclusions: Data suggest that the effects of transient silencing on cell proliferation differ depending on the initial cell seeding density. While high seeding densities mask the significant changes in proliferation, the inhibitory effects of silencing become apparent at lower seeding densities as the entry into log phase is delayed. Using the optimal initial seeding density is crucial when studying the effects of transient gene silencing. In addition, the results suggest that TRPC1 may contribute to proliferation and phenotypic switching of vascular smooth muscle cells.
\end{abstract}

Keywords: A7r5, Cell density, Huh7, Impedance, RTCA

\section{Background}

Impedance-based real-time cellular analysis (RTCA) systems enable label-free, non-invasive and kinetic monitoring of cellular events in contrast to labour-intensive label-based end-point measurements [1]. The xCELLigence RTCA system, developed by Roche Applied Science (Penzberg, Germany) and currently marketed by the original inventor ACEA Biosciences (San Diego, USA), allows dynamic monitoring of various processes

\footnotetext{
*Correspondence: cigdemselli@gmail.com

${ }^{1}$ Applied Bioinformatics of Cancer, Edinburgh Cancer Research

Centre, Institute of Genetics and Molecular Medicine, The University

of Edinburgh, Crewe Road South, Edinburgh EH4 2XR, UK

Full list of author information is available at the end of the article
}

including proliferation, migration and invasion [2]. The system is also a powerful and reliable drug discovery tool for toxicological and pharmacological studies [3] including cardiovascular safety testing [4-7] and drug screening [8-11]. Although xCELLigence system is originally designed to work with adherent cells, successful monitoring of non-adherent cells such as cells from haematological malignancies by pre-coating of the cell culture surface with specific adhesive substrates [12] has expanded its area of use.

Since each cell type has its own characteristic growth pattern, the optimum seeding concentration of each cell type gives a short lag period and early onset of logarithmic growth should be determined before the 
proliferation assays using RTCA system. For this purpose, performing preliminary experiments to obtain the cell growth patterns at different seeding densities is suggested (xCELLigence Application Note No.7/January 2009). We proposed that the optimum cell seeding density also depends on each experimental condition and the actual experiments should also be performed at different seeding densities in addition to the preliminary experiments, especially for transiently-silenced cells.

Following its discovery in mammalian cells [13], RNA interference has been used as a powerful tool to study gene function by administration of small interfering RNA (siRNA) and short hairpin RNA (shRNA) [14]. A significant disadvantage of siRNA application is that its concentration becomes diluted as the cells divide resulting in transient silencing. It is possible to generate longterm knockdown of the gene of interest by integration of shRNA into the host genome [15]; however, the creation of a stable shRNA cell line is time-consuming and may take months. Since stable knockdown requires longer subculturing process, it was not applicable to A7r5 cells because subculturing leads to alterations in A7r5 cell phenotype and proliferation [16]. Therefore, in the present study, we performed transient silencing of transient receptor potential canonical 1 (TRPC1) levels in A7r5 cells.

Although still controversial, TRPC1 has been suggested to be an essential component of store-operated $\mathrm{Ca}^{2+}$ (SOC) entry channels in heteromultimeric combinations with other TRPCs $[17,18]$. SOC entry, a mechanism activated by emptying of intracellular $\mathrm{Ca}^{2+}$ stores [19], was suggested to maintain optimal sarcoendoplasmic reticulum $\mathrm{Ca}^{2+}$ levels mediating $\mathrm{Ca}^{2+}$ signalling-related cellular processes including proliferation [20]. We previously demonstrated reciprocal changes in TRPC1 and TRPC6 levels in A7r5 vascular smooth muscle cells [21] and in aging rat thoracic aorta [22]. Furthermore, downregulation of TRPC1 significantly elevated SOC entry suggesting the regulatory role of TRPC1 both in A7r5 [21] and Huh7 hepatocellular carcinoma cells [23]. TRPC1 silencing also suppresses Huh7 cell proliferation without affecting cell migration in real-time cellular analyses suggesting the role of TRPC1 in the regulation of hepatocellular carcinoma cell proliferation [23].

Based on these data, we monitored the real-time changes in proliferation of Huh7 and A7r5 cells with different seeding densities following transient TRPC1 gene silencing using E-plate 96 and xCELLigence MP system.

\section{Methods}

\section{Cell culture}

Human hepatocellular carcinoma cell line, Huh7, cultured in DMEM (Biological Industries, Cromwell,
USA) supplemented with $10 \%$ fetal bovine serum (FBS, HyClone, Logan, USA), $2 \mathrm{mM}$ L-glutamine (HyClone, Logan, USA) and $0.1 \mathrm{mM}$ non-essential amino acid solution (Gibco, Waltham, USA). Vascular smooth muscle cell line (A7r5, an immortalized line derived from embryonic rat aorta) cultured in DMEM/Ham's F12 (Gibco, Waltham, USA) supplemented with $10 \%$ FBS (Gibco, Waltham, USA) and $2 \mathrm{mM} \mathrm{L-glutamine}$ (Gibco, Waltham, USA). Cells were maintained in a humidified incubator at $37{ }^{\circ} \mathrm{C}$ and $5 \% \mathrm{CO}_{2}$ and were subcultured using $0.5 \%$ trypsin-EDTA when reached $70 \%$ confluency. Huh7 and A7r5 cells were subcultured with 1:2 and 1:3-1:4 split ratios, respectively, and passage numbers $(\mathrm{P} \#)$ were recorded. Regular checks for mycoplasma contamination were performed using MycoAlert Mycoplasma Detection kit (Lonza, Basel, Switzerland). After freezing in feeding medium with $5 \%$ DMSO, cells were stored in the vapour phase of liquid nitrogen. A7r5 cell line purchased at $\mathrm{P} \# 11$ from the American Type Culture Collection (ATCC; CRL1444). Huh7 cells, originally from Jack Wands Laboratory at Massachusetts General Hospital, Boston, MA, were kindly provided by Professor Mehmet Ozturk (Dokuz Eylul University, Izmir, Turkey), considered to be at their first passage ( $\mathrm{P} \# 1$ ) at the time of arrival to our laboratory and were tested for authenticity in 2010 .

\section{Real-time monitoring of proliferation}

Real-time monitoring of cell proliferation performed using xCELLigence MP system. E-plate 96, used with xCELLigence system, is a single-use 96 well cell culture plate with bottom surfaces covered with microelectrode sensors $\left(0.2 \mathrm{~cm}^{2}\right.$ well surface area; $243 \pm 5 \mu \mathrm{l}$ maximum volume). Real-time changes in electrical impedance measured using the gold microelectrodes and expressed as "cell index" defined as $(\mathrm{Rn}-\mathrm{Rb}) / 15$, where $\mathrm{Rb}$ is the background impedance and $\mathrm{Rn}$ is the impedance of the well with cells. Negative control groups (wells containing $200 \mu \mathrm{l}$ culture medium without cells with cell index values around 0) were tested in every experiment; however, they were not shown in figures in order to simplify the representations.

Before seeding cells into E-plate 96, the background impedance was measured after the addition of $100 \mu \mathrm{l}$ medium and a 30 min-incubation period at room temperature. Cell density was determined by using a haemocytometer after methylene blue staining. Following the seeding of the appropriate number of cells into the wells, the plate incubated at room temperature for $30 \mathrm{~min}$ in order to allow cell settling. Cell proliferation monitored every $30 \mathrm{~min}$ for $72 \mathrm{~h}$. Cells were fed with $200 \mu \mathrm{l}$ well $^{-1}$ fresh medium every $48 \mathrm{~h}$. 


\section{Transient TRPC1 gene silencing}

In silencing experiments, Huh7 cells were transfected in 6-well plates with pSUPERIOR.shTRPC1, or empty vector as negative control, as described previously [23]. Briefly, to construct pSUPERIOR.shTRPC1 vector, TRPC1 silencing shRNA sequence (shTRPC1, with a 19 nucleotide-silencing sequence which targets 361-379th nucleotides of TRPC1 mRNA) purchased and cloned into pSUPERIOR.retro. neo + gfp vector (Oligoengine, Seattle, USA). After $48 \mathrm{~h}$ vector incubation, Huh7 cells were seeded into E-plate 96 at different densities $\left(10,000,5000\right.$ and 1250 cells well $\left.^{-1}\right)$.

In addition, A7r5 cells transfected with $2 \mu \mathrm{g}$ pSUPERIOR.shTRPC1 or empty vector as negative control via $6 \mu \mathrm{l}$ FugeneHD transfection reagent (Roche Applied Science, Penzberg, Germany) were seeded into E-plate 96 at different densities (5000 and 2500 cells well $^{-1}$ ) $48 \mathrm{~h}$ after the vector incubation.

\section{Quantitative real-time RT-PCR}

Effects of vector transfection on TRPC1 expression levels in A7r5 cells were measured by quantitative realtime RT-PCR using FastStart DNA Master SYBR Green I kit and LightCycler 1.5 (Roche Applied Science, Penzberg, Germany). High Pure RNA Isolation Kit (Roche Applied Science, Penzberg, Germany) and Dynamo cDNA Synthesis Kit (Finnzymes, Waltham, USA) used to perform total RNA isolation and reverse transcription, respectively. Primers used for TRPC1 (NM_053558) and beta-actin (NM_031144) were as follows: forward 5"TGGTATGAAGGGTTGGAAGAC'3 [24], reverse 5"TGCTGTTCACAGAAGATGCC'3 [25], and forward 5'AGTGTGACGTTGACATCCGT'3 [26], reverse 5'GACTCATCGTACTCCTGCTT'3 [26]. TRPC1 expression levels were normalized to that of internal $\beta$-actin and expressed as [TRPC $1 / \beta$-actin $\times 100]$.

\section{Data analysis}

Data expressed as mean \pm standard deviation. "n" represents the number of samples. Statistical significance between the means of two groups was evaluated using Student's $t$ test, with $p<0.05$ considered significant. Data analyses and graphical presentations performed using GraphPad Prism 5 (La Jolla, USA).

\section{Results}

\section{Effects of cell seeding density on Huh7 proliferation following TRPC1 silencing}

In order to determine the effects of seeding density on antiproliferative effects of transient gene silencing, real-time changes in proliferation were monitored in TRPC1-silenced Huh7 cells seeded at different densities into E-plate 96. Huh7 cells transfected with silencing vector that also emit GFP signal was observed using an inverted fluorescent microscope (Olympus IX71) and the transfection efficiency was determined (70\%) by monitoring GFP fluorescence emission at $520 \mathrm{~nm}$ with excitation at $460-490 \mathrm{~nm}$ (Fig. 1a). Huh7 cells were also observed on E-plate 96 at the end of each experiment. Although the non-transparent gold microelectrodes preclude the accurate prediction of the transfection efficiency, an apparent decrease in fluorescence signal was monitored due to dilution of transfected cells as the cells divide, as expected (Fig. 1b). The company (ACEA Biosciences, San Diego, USA) is currently marketing modified version of plates with a small opening in the electrode array, E-Plate VIEW, allowing the visual inspection the cells under an inverted microscope.

TRPC1 silencing did not inhibit the proliferation of Huh7 cells at 10,000 cells well ${ }^{-1}$ seeding density $(n=10$, Fig. 2a). When seeded at 5000 cells well $^{-1}$, proliferation was significantly suppressed for the first $24 \mathrm{~h}(p<0.01$; $\mathrm{n}=9$; Fig. 2b). Both control and silenced-cells reached plateau cell index after $30 \mathrm{~h}$ at 10,000 cells well $^{-1}$ seeding density. At 5000 cells well $^{-1}$ density, control and silenced-cells reached plateau cell index after 36 and $42 \mathrm{~h}$, respectively. After these steady-state time points, significant but incoherent increases in proliferation were observed at both seeding densities $(p<0.01$; Fig. 2a, b). However, TRPC1 silencing significantly inhibited the proliferation of Huh7 cells compared to control cells in 1250 cells well $^{-1}$ seeding density at each time point for $72 \mathrm{~h}(p<0.01 ; \mathrm{n}=12$; Fig. 2c).

\section{Effects of cell seeding density on A7r5 proliferation following TRPC1 silencing}

A7r5 proliferation curves at four different seeding densities $\left(10,000-1250\right.$ cells well $\left.^{-1}\right)$ were monitored before determining the effects of silencing on proliferation. Cell index increased proportionally to A7r5 cell densities as expected (Fig. 3a). Cells reached a plateau after 24 and $48 \mathrm{~h}$ at 10,000 and 5000 cells well $^{-1}$ densities, respectively. Cells at 2500 and 1250 cells well $^{-1}$ seeding densities showed exponential increases in proliferation and did not reach the plateau after $72 \mathrm{~h}$.

The effects of TRPC1 silencing on expression levels were determined using real-time qRT-PCR before performing the proliferation assay. TRPC1 silencing significantly inhibited the TRPC1 mRNA expression levels compared to control cells $(p<0.05, \mathrm{n}=3$, Fig. $3 \mathrm{~b})$. Although the silencing partially (29\%) inhibited TRPC1 mRNA levels, its inhibitory effect on cell proliferation was significant.

Based on the proliferation curves previously performed to determine the optimum cell density (Fig. 3a), real-time changes in proliferation were monitored at 5000 and 2500 cells well $^{-1}$ seeding densities in TRPC1silenced A7r5 cells. TRPC1 silencing significantly 


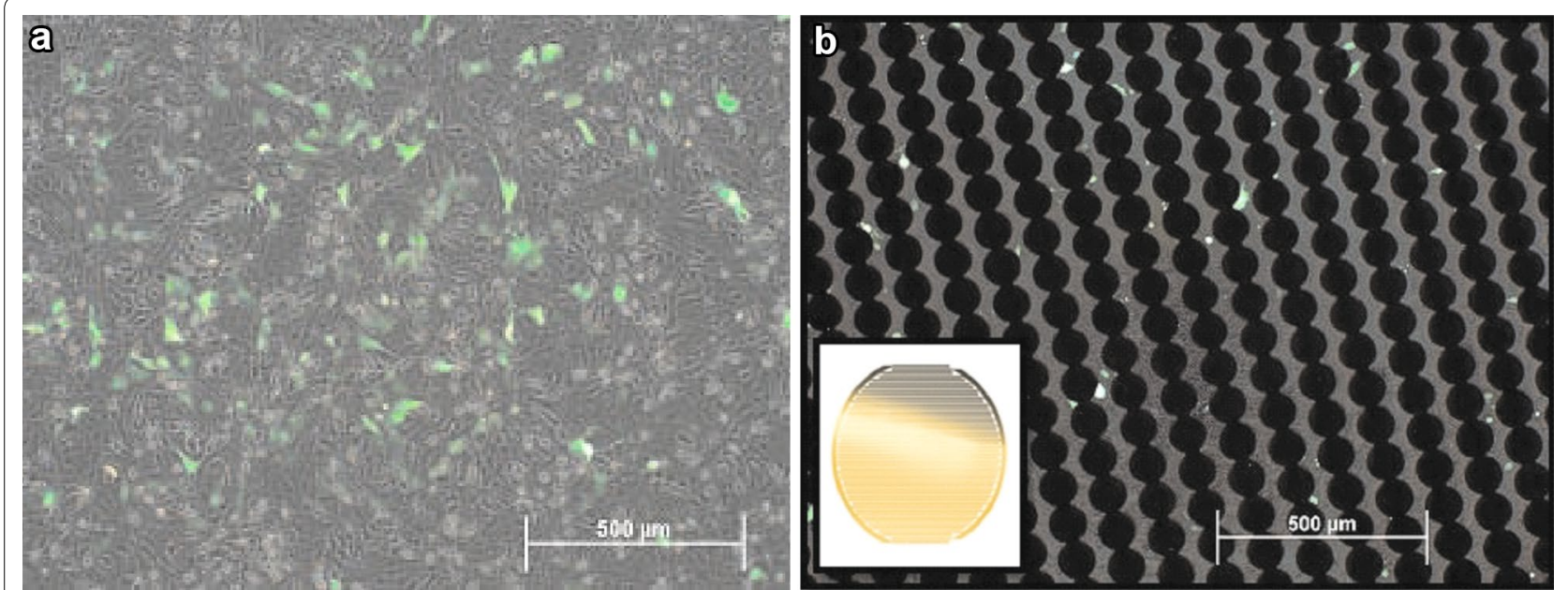

Fig. 1 Fluorescent microscopy images of Huh7 cells. a Silencing vector-transfected Huh7 cells were observed in a 6-well plate after $48 \mathrm{~h}$ incubation before seeding into E-plate 96. b At the end of the proliferation assay, Huh7 cells on E-plate 96 were visible between microelectrodes seen as aligned closed circles. The insert represents the gold microelectrode sensors covering the bottom surface of the well (from the manufacturer's manual)

inhibited the proliferation of A7r5 cells at both 5000 and 2500 cells well $^{-1}$ seeding densities for $72 \mathrm{~h}(p<0.01$, $\mathrm{n}=8$, Fig. 4). Both control and silenced cells did not reach the plateau cell index at either seeding densities.

\section{Discussion}

Since the initial seeding density is critical in functional tissue engineering, the proliferation of human umbilical cord mesenchymal stem cells (hUCMSCs) seeded on calcium phosphate cement, a scaffold material used for bone tissue engineering, at different densities has been investigated previously [27]. Zhou et al. showed that hUCMSC proliferation and osteodifferentiation increased proportional to cell seeding density from 50,000 to the optimum value of 300,000 (cells in a 24-well plate), with a decrease over this limit [27]. Furthermore, to determine the endothelial cellbiomaterial interaction that had an impact on the development of biomedical implants, the effects of cell-seeding density on the proliferation rate of human umbilical vein endothelial cells (HUVEC) seeded on different biomaterials including tissue culture polystyrene were investigated [28]. Maximal HUVEC proliferation was obtained at an initial seeding density of 1000 cells $\mathrm{cm}^{-2}$ with a sharp decrease both above and below of this particular density [28].

Although cell proliferation is required for physiological processes such as renewal of intestinal epithelium [29] and wound healing [30], its abnormalities are associated with various diseases such as tumorigenesis [31]. The involvement of TRPC1 in cell proliferation in different types of cancers [32-34] as well as in endothelial progenitor cells [35] has been previously reported. In addition, Huh7 cell line was shown to have a subpopulation of cells with hepatic cancer stem cell-like properties that express alpha-fetoprotein and epithelial cell adhesion molecule (EPCAM), a hepatic stem cell biomarker [32].

In our previous study, inhibitory effects of silencing vector administration on TRPC1 expression levels in Huh7 cells were determined by quantitative real-time RT-PCR and western blot analyses [23]. After TRPC1 silencing vector transfection, mRNA levels were shown to be inhibited reversibly with a significant decrease at $48 \mathrm{~h}$ and recovered at $72 \mathrm{~h}$ [23]. Furthermore, we observed significant suppression in proliferation rates and increase in doubling time in TRPC1-silenced Huh7 cells at 2500 cells well $^{-1}$ seeding density [23]. Based on these data, among the hepatocellular carcinoma cell lines, Huh7 cells were chosen to further study the effects of cell seeding density on the proliferation of TRPC1-silenced cells. Inhibitory effects of transient TRPC1 silencing on Huh7 proliferation rates were masked at 10,000 cells well ${ }^{-1}$ seeding density with a significant decrease only after $24 \mathrm{~h}$ at 5000 cells well $^{-1}$ seeding density. Moreover, the effects of silencing were much more significant at 1250 cells well ${ }^{-1}$ seeding density at each time point throughout the whole assay suggesting

Fig. 2 Real-time proliferation of TRPC1-silenced Huh7 (P\# 26) cells at different densities. At $48 \mathrm{~h}$ following shTRPC1 transfection, cells were seeded on E-plate 96 and cell proliferation monitored real-time for 3 days. The proliferation curves and cumulative data of cells at different densities (cells well ${ }^{-1}$ ); a 10,000, b 5000 and $\mathbf{c} 1250$ are shown ${ }^{* *} p<0.01, n=10, n=9$ and $n=12$, respectively) 

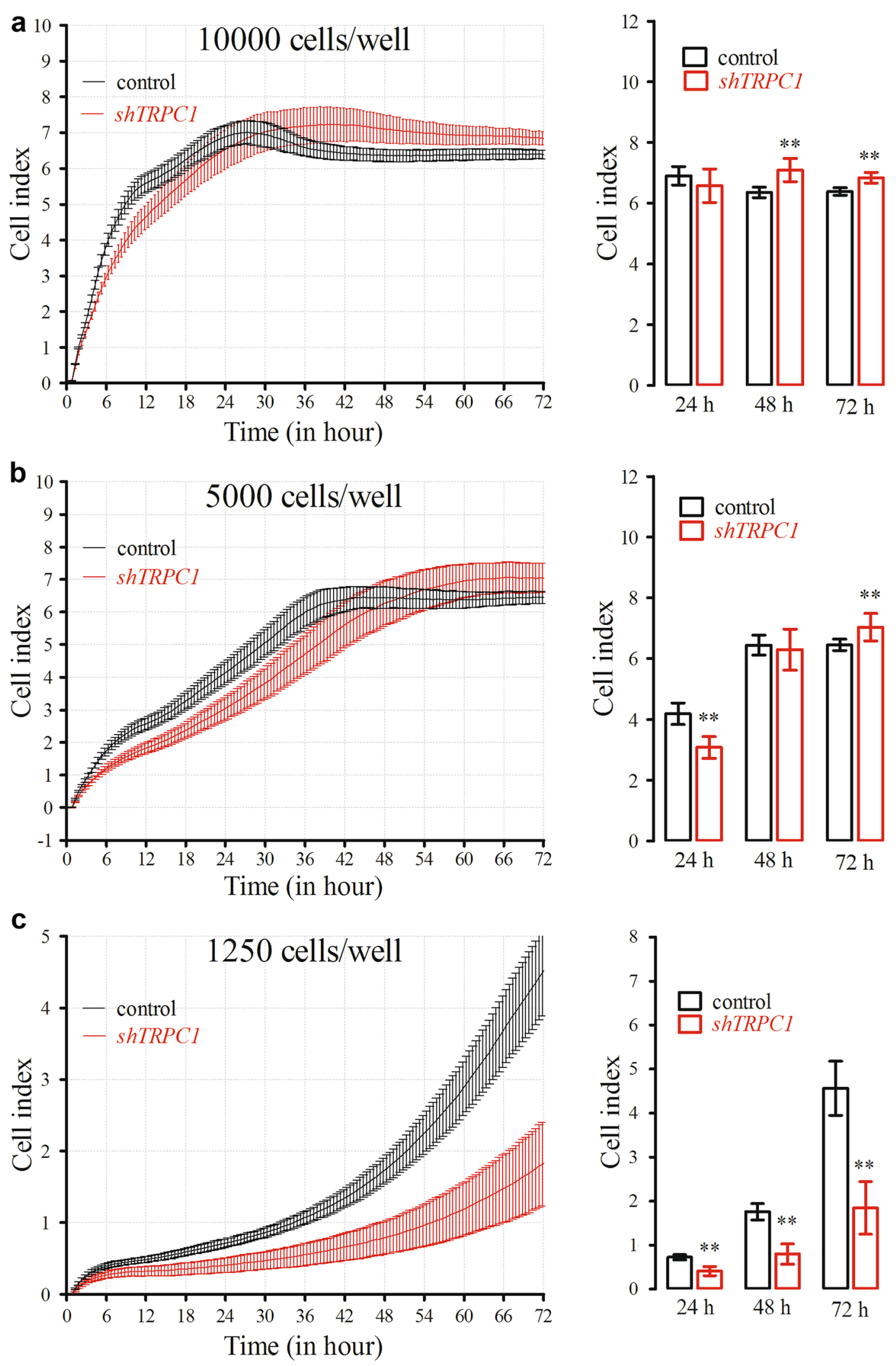

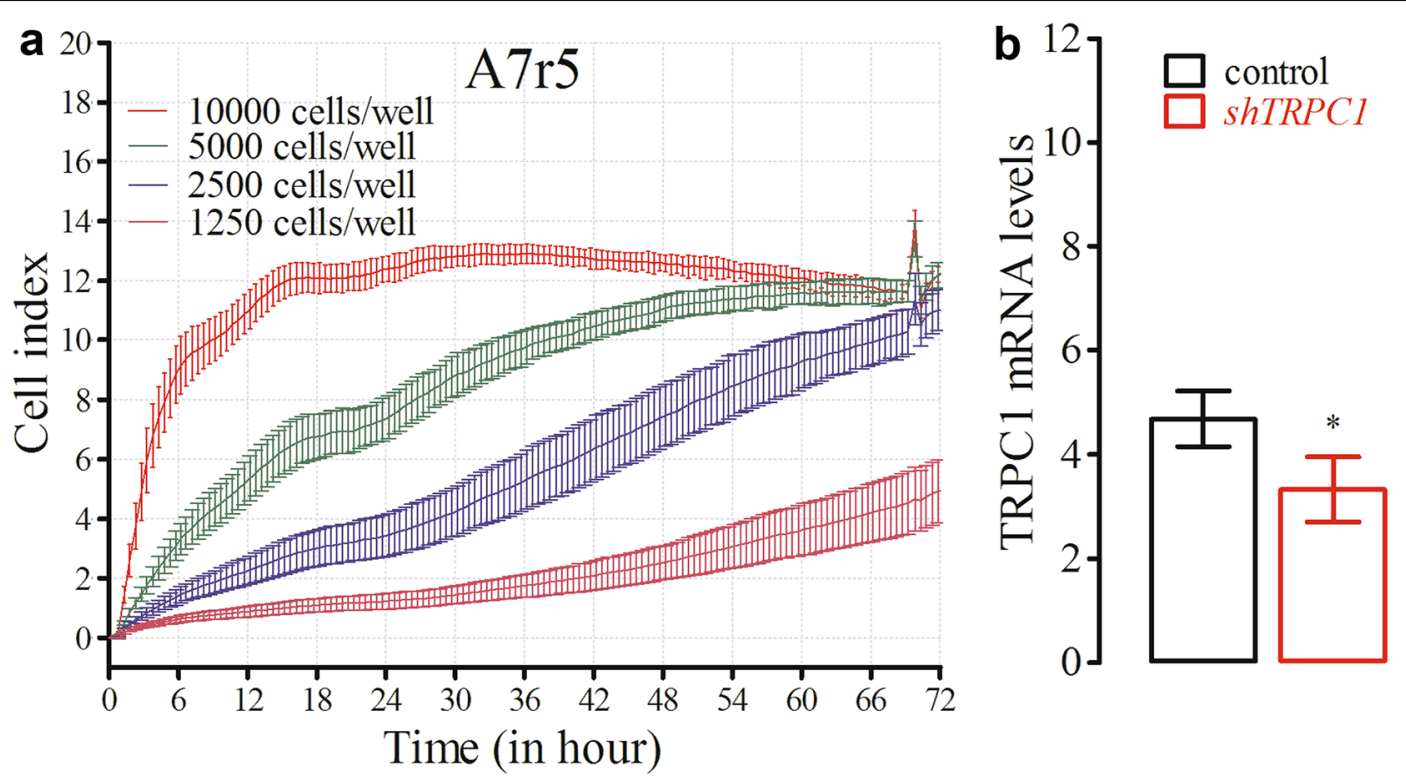

Fig. 3 Real-time proliferation curves and cumulative mRNA expression data of A7r5 cells. The proliferation of A7r5 cells (P\# 17) at different cell densities were monitored for $72 \mathrm{~h}(\mathbf{a} ; n=8)$. TRPC1 mRNA expression levels were determined $72 \mathrm{~h}$ following silencing vector transfection $\left(\mathbf{b},{ }^{*} p<0.05\right.$, $n=3)$

the monitoring cells in very early points of log period as well as before reaching the plateau phase is essential to detect the effects of transient silencing. Therefore, the seeding density that allows cells to reach the plateau levels after $72 \mathrm{~h}$ or later should be used for Huh7 cells.

In addition to cancer cells, we also monitored the effects of seeding density on the proliferation of TRPC1-silenced A7r5 cells which has a significance in examining the vascular contractile and proliferative phenotypes in vitro [36]. It is known that vascular smooth muscle cells have the ability of plasticity with a wide range of phenotypes besides their primary contractile phenotype [37]. Evidence suggests that switching from contractile to proliferating (non-contractile/synthetic) phenotype is associated with vascular diseases [38, 39]. Differential expression of calcium handling proteins including upregulated TRPC1 and TRPC6 expression levels were associated with the proliferative phenotype $[39,40]$. In our previous study investigating the effects of $\mathrm{A} 7 \mathrm{r} 5$ passaging that may mimic the phenotypic switching of vascular smooth muscle cells, we observed upregulated SERCA2b mRNA and SOC entry levels along with suppression of proliferation [16]. In the present study, knockdown of TRPC1 inhibited A7r5 cell proliferation suggesting its possible contribution to vascular smooth muscle cell proliferation and phenotypic switching. Furthermore, the inhibitory effect of transient TRPC1 silencing on A7r5 proliferation was observed at both seeding densities tested in our study (5000 and 2500 cells well $^{-1}$, respectively) suggesting that the seeding density that allows cells to reach the plateau levels after $72 \mathrm{~h}$ or later is optimal for studying the effects of transient silencing on A7r5 cells.

In silencing experiments, at least $72 \mathrm{~h}$ incubation is required to observe the suppression of target protein and resulting phenotypic alterations. We have previously shown that TRPC1 mRNA levels were inhibited reversibly with a significant decrease at $48 \mathrm{~h}$ and recovered at $72 \mathrm{~h}$ in Huh7 cells [23]. In addition, TRPC1 protein levels decreased significantly at $72 \mathrm{~h}$ after silencing vector transfection in A7r5 and Huh7 cells [23]. In the current study, cells were seeded on E-plate 96 after $48 \mathrm{~h}$ vector incubation and therefore, at least a 24 -h period is required to observe an effect on protein levels and proliferation. When cells were seeded in higher densities, they reached plateau proliferation levels at $24 \mathrm{~h}$ which may limit the production of siRNA. Significant changes in proliferation were observed if cells were seeded in optimum densities possibly allowing sufficient time to downregulate target protein levels. Determination of mRNA and protein levels $24 \mathrm{~h}$ following seeding on E-plates at different densities requires further investigation.

\section{Conclusions}

The effects of transient silencing on cell proliferation vary depending on the cell seeding density at the start of the RTCA experiments. Higher seeding densities mask the significant changes in proliferation rates whereas they become clear at lower seeding densities as the log phase is delayed. Therefore, determination of optimal cell seeding density for real-time monitoring of proliferation in 

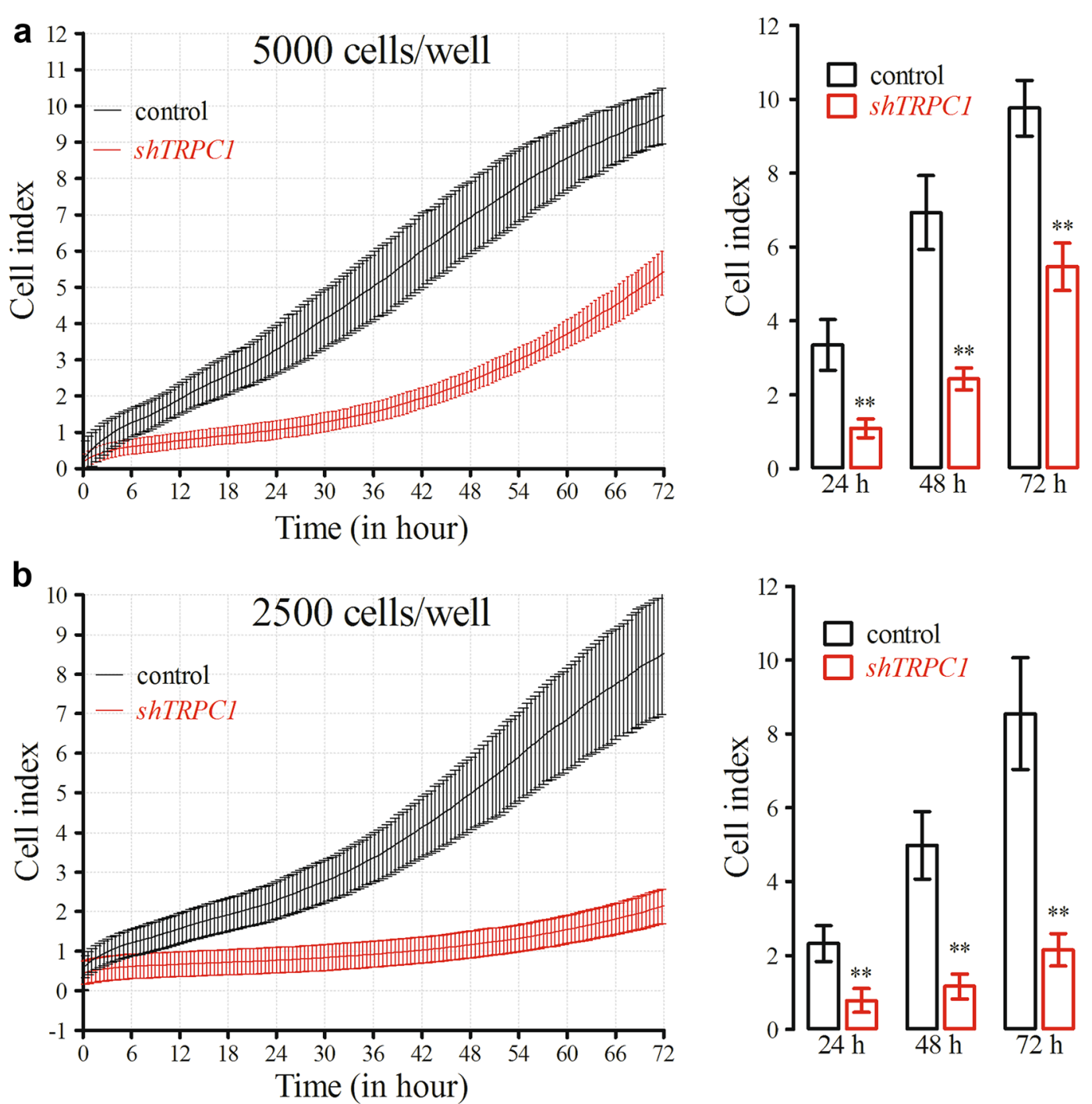

Fig. 4 Real-time proliferation of TRPC1-silenced A7r5 cells (P\# 20) at different cell densities. At $48 \mathrm{~h}$ following shTRPC1 transfection, 5000 (a) and 2500 (b) cells well ${ }^{-1}$ were seeded on E-plate 96 and cell proliferation rates were monitored real-time for 3 days $\left({ }^{* *} p<0.01, n=8\right)$

transiently-silenced cells is crucial for more accurate data acquisition and evaluation. We suggest that real-time monitoring studies should be performed using a range of cell type specific initial seeding densities in order to determine the effects of transient gene-knockdown. This would help to improve our knowledge about the outcome of transient gene silencing. Beyond the technical aspects, the data suggest that TRPC1 may contribute to proliferation and phenotypic switching of vascular smooth muscle cells.

\section{Authors' contributions}

CS designed and carried out the experiments, analyzed the data, wrote and completed the manuscript. YE helped to design and perform the experiments and analyzed the data. MT supervised the experiments. All authors read, edited and approved the final manuscript. All authors read and approved the final manuscript

\section{Author details}

${ }_{1}^{1}$ Applied Bioinformatics of Cancer, Edinburgh Cancer Research Centre, Institute of Genetics and Molecular Medicine, The University of Edinburgh, Crewe Road South, Edinburgh EH4 2XR, UK. ${ }^{2}$ Department of Pharmacology, Faculty of Pharmacy, Ege University, 35040 Izmir, Turkey. ${ }^{3}$ Faculty of Medicine, Izmir University of Economics, 35330 Izmir, Turkey.

\section{Acknowledgements}

The authors would like to thank Professors Mehmet Ozturk and Esra S. Erdal for providing cells.

\section{Competing interests}

The authors declare that they have no competing interests.

\section{Availability of data and materials}

The datasets analyzed during the current study available from the corresponding author on reasonable request. 


\section{Funding}

This work was supported by The Scientific and Technological Research Council of Turkey (TUBITAK Research Project, 108S072) and Novartis (Turkey) to MT. The xCELLigence system was purchased within the Research Infrastructure Project, The State Planning Organization of Turkey (DPT, 2009K120640).

Received: 2 July 2016 Accepted: 25 November 2016 Published online: 01 December 2016

\section{References}

1. Limame R, Wouters A, Pauwels B, Fransen E, Peeters M, Lardon F, et al. Comparative analysis of dynamic cell viability, migration and invasion assessments by novel real-time technology and classic endpoint assays. PLOS ONE. 2012;7:e46536.

2. Atienza JM, Yu NC, Kirstein SL, Xi B, Wang X, Xu X, et al. Dynamic and label-free cell-based assays using the real-time cell electronic sensing system. Assay Drug Dev Technol. 2006:4:597-607.

3. Atienzar FA, Gerets H, Tilmant K, Toussaint G, Dhalluin S. Evaluation of impedance-based label-free technology as a tool for pharmacology and toxicology investigations. Biosensors. 2013;3:132-56.

4. Xi B, Wang T, Li N, Ouyang W, Zhang W, Wu J, et al. Functional cardiotoxicity profiling and screening using the xCELLigence RTCA Cardio System. J Lab Autom. 2011;16:415-21.

5. Peters MF, Scott CW, Ochalski R, Dragan YP. Evaluation of cellular impedance measures of cardiomyocyte cultures for drug screening applications. Assay Drug Dev Technol. 2012;10:525-32.

6. Himmel HM. Drug-induced functional cardiotoxicity screening in stem cell-derived human and mouse cardiomyocytes: effects of reference compounds. J Pharmacol Toxicol Methods. 2013:68:97-111.

7. Peters MF, Lamore SD, Guo L, Scott CW, Kolaja KL. Human stem cellderived cardiomyocytes in cellular impedance assays: bringing cardiotoxicity screening to the front line. Cardiovasc Toxicol. 2015;15:127-39.

8. Atienza JM, Yu N, Wang X, Xu X, Abassi Y. Label-free and real-time cellbased kinase assay for screening selective and potent receptor tyrosine kinase inhibitors using microelectronic sensor array. J Biomol Screen. 2006;11:634-43.

9. Smout MJ, Kotze AC, MCCarthy JS, Loukas A. A novel high throughput assay for anthelmintic drug screening and resistance diagnosis by realtime monitoring of parasite motility. PLoS Negl Trop Dis. 2010;4:e885.

10. Hou XQ, Yan R, Yang C, Zhang L, Su RY, Liu SJ, et al. A novel assay for highthroughput screening of anti-Alzheimer's disease drugs to determine their efficacy by real-time monitoring of changes in PC12 cell proliferation. Int J Mol Med. 2014;33:543-9.

11. Kho D, MacDonald C, Johnson R, Unsworth CP, O'Carroll SJ, du Mez E et al. Application of xCELLigence RTCA biosensor technology for revealing the profile and window of drug responsiveness in real time. Biosensors. 2015;5:199-222.

12. Martinez-Serra J, Gutierrez A, Muñoz-Capó S, Navarro-Palou M, Ros T, Amat JC, et al. xCELLigence system for real-time label-free monitoring of growth and viability of cell lines from hematological malignancies. Onco Targets Ther. 2014;7:985-94.

13. Fire A, Xu S, Montgomery MK, Kostas SA, Driver SE, Mello CC. Potent and specific genetic interference by double-stranded RNA in Caenorhabditis elegans. Nature. 1998;391:806-11.

14. Moore CB, Guthrie EH, Huang MT, Taxman DJ. Short hairpin RNA (shRNA): design, delivery, and assessment of gene knockdown. Methods Mol Biol. 2010;629:141-58

15. Paddison PJ, Caudy AA, Hannon GJ. Stable suppression of gene expression by RNAi in mammalian cells. Proc Natl Acad Sci USA. 2002;99:1443-8.

16. Erac $Y$, Selli C, Filik P, Tosun M. Effects of passage number on proliferation and store-operated calcium entry in A7r5 vascular smooth muscle cells. J Pharmacol Toxicol Methods. 2014;70:1-5.

17. Liu X, Singh BB, Ambudkar IS. TRPC 1 is required for functional storeoperated $\mathrm{Ca}^{2+}$ channels. Role of acidic amino acid residues in the S5-S6 region. J Biol Chem. 2003;278:11337-43.
18. Ambudkar IS, Ong HL, Liu X, Bandyopadhyay BC, Cheng KT. TRPC1: the link between functionally distinct store-operated calcium channels. Cell Calcium. 2007:42:213-23.

19. Putney JW Jr. A model for receptor-regulated calcium entry. Cell Calcium. 1986;7:1-12.

20. Venkatachalam K, van Rossum DB, Patterson RL, Ma HT, Gill DL. The cellular and molecular basis of store-operated calcium entry. Nat Cell Biol. 2002;4:E263-72.

21. Selli C, Erac Y, Kosova B, Tosun M. Post-transcriptional silencing of TRPC1 ion channel gene by RNA interference upregulates TRPC6 expression and store-operated $\mathrm{Ca}^{2+}$ entry in A7r5 vascular smooth muscle cells. Vasc Pharmacol. 2009;51:96-100.

22. Erac Y, Selli C, Kosova B, Akcali KC, Tosun M. Expression levels of TRPC1 and TRPC6 ion channels are reciprocally altered in aging rat aorta: implications for age-related vasospastic disorders. Age. 2010;32:223-30.

23. Selli C, Erac Y, Kosova B, Erdal ES, Tosun M. Silencing of TRPC1 regulates store-operated calcium entry and proliferation in Huh7 hepatocellular carcinoma cells. Biomed Pharmacother. 2015:71:194-200.

24. Flemming R, Xu SZ, Beech DJ. Pharmacological profile of store-operated channels in cerebral arteriolar smooth muscle cells. Br J Pharmacol. 2003;139:955-65

25. Kohler R, Brakemeier S, Kühn M, Degenhardt C, Buhr H, Pries A, et al. Expression of ryanodine receptor type 3 and TRP channels in endothelial cells: comparison of in situ and cultured human endothelial cells. Cardiovasc Res. 2001;51:160-8.

26. Wang J, Shimoda LA, Sylvester JT. Capacitative calcium entry and TRPC channel proteins are expressed in rat distal pulmonary arterial smooth muscle. Am J Physiol Lung Cell Mol Physiol. 2004;286:L848-58.

27. Zhou HZ, Weir MD, Xu HH. Effect of cell seeding density on proliferation and osteodifferentiation of umbilical cord stem cells on calcium phosphate cement-fiber scaffold. Tissue Eng Part A. 2011;17:2603-13.

28. Heng BC, Bezerra PP, Preiser PR, Law SKA, Xia Y, Boey F, et al. Effect of cell-seeding density on the proliferation and gene expression profile of human umbilical vein endothelial cells within ex vivo culture. Cytotherapy. 2011;13:606-17.

29. Podolsky DK. Regulation of intestinal epithelial proliferation: a few answers, many questions. Am J Physiol. 1993;264:G179-86.

30. Velnar T, Bailey T, Smrkolj V. The wound healing process: an overview of the cellular and molecular mechanisms. J Int Med Res. 2009;37:1528-42.

31. Hanahan D, Weinberg RA. Hallmarks of cancer: the next generation. Cell. 2011;144:646-74

32. Yamashita T, Ji J, Budhu A, Forgues M, Yang W, Wang HY, et al. EpCAMpositive hepatocellular carcinoma cells are tumor-initiating cells with stem/progenitor cell features. Gastroenterology. 2009;136:1012-24.

33. Bomben VC, Sontheimer H. Disruption of transient receptor potential canonical channel 1 causes incomplete cytokinesis and slows the growth of human malignant gliomas. Glia. 2010;58:1145-56.

34. Tajeddine N, Gailly P. TRPC1 protein channel is major regulator of epidermal growth factor receptor signaling. J Biol Chem. 2012;287:16146-57.

35. Kuang CY, Yu Y, Wang K, Qian DH, Den MY, Huang L. Knockdown of transient receptor potential canonical-1 reduces the proliferation and migration of endothelial progenitor cells. Stem Cells Dev. 2012;21:487-96.

36. Kennedy E, Hakimjavadi R, Greene C, Mooney CJ, Fitzpatrick E, Collins LE, et al. Embryonic rat vascular smooth muscle cells revisited - a model for neonatal, neointimal SMC or differentiated vascular stem cells? Vasc Cell. 2014;6:6.

37. Yoshida T, Owens GK. Molecular determinants of vascular smooth muscle cell diversity. Circ Res. 2005;96:280-91.

38. Owens GK, Kumar MS, Wamhoff BR. Molecular regulation of vascular smooth muscle cell differentiation in development and disease. Physiol Rev. 2004;84:767-801.

39. House SJ, Potier M, Bisaillon J, Singer HA, Trebak M. The non-excitable smooth muscle: calcium signaling and phenotypic switching during vascular disease. Pflugers Arch. 2008:456:769-85.

40. Bergdahl A, Gomez MF, Wihlborg A-K, Erlinge D, Eyjolfson A, Xu S-Z, et al. Plasticity of TRPC expression in arterial smooth muscle: correlation with store-operated Ca ${ }^{2+}$ entry. Am J Physiol Cell Physiol. 2005;288:C872-80. 